\title{
Dopo la Via della Seta e prima delle Nuove Vie della Seta \\ Il viaggio in Cina \\ di Carla Novellis di Coarazze (1906-1907)
}

\section{Stefano Piastra}

Alma Mater Studiorum Università di Bologna

DOI: http://dx.doi.org/10.7358/gn-2018-001-pias

\section{AbSTRACT}

The paper analyzes geo-historical, topographical and perceptive issues of the travel diary by Carla Novellis di Coarazze (1865-?), née Dreyfus, of Hungarian origin, in China from November 1906 to January 1907 together with her husband, Italian Baron Carlo Maria Novellis di Coarazze (1855-1916), commander on board of the cruiser Marco Polo of the Italian Royal Navy. This travelogue, still unpublished, is now conserved in the Italian National Diary Archive, based in Pieve Santo Stefano (Province of Arezzo). In the framework of her Chinese stay, Carla Novellis describes mostly the quasi-colonial environment of the 'Old Shanghai' at the beginning of the 20th century, but also Suzhou and Guangzhou. The paper georeferences and discusses places of the above mentioned cities, quoted by Novellis in her diary.

Keywords: Carla Novellis di Coarazze; China; cultural geography; travelogues.

Parole chiave: Carla Novellis di Coarazze; Cina; geografia culturale; racconti di viaggio.

1. LE COORDINATE GEOSTORICHE DI LUNGO PERIODO DEL VIAGGIO dall’Europa alla Cina: da Marco Polo

a Carla Novellis di Coarazze

A cavallo fra XIII e XIV secolo, la dinastia mongola Yuan allora regnante sulla Cina garantì, nell'ambito di quella che è nota come pax mongolica, comunicazioni terrestri relativamente stabili e sicure tra Europa e Asia orien- 
tale attraverso le steppe e i deserti centro-asiatici. La merce più preziosa che veniva veicolata lungo tali direttrici, ovvero la seta, fu alla base della denominazione dell'itinerario medesimo, proposta da Ferdinand von Richthofen negli anni Settanta del XIX secolo: lungo la Via della Seta si mossero quindi sia mercanti (il più famoso fu il veneziano Marco Polo, ma negli stessi anni risultavano attestati anche commercianti genovesi: Bertuccioli e Masini 1996) sia, in seguito al clima di tolleranza religiosa promosso dagli Yuan, missionari. Questi ultimi furono in primis francescani, da Giovanni da Pian del Carpine, a Guglielmo di Rubruquis, Giovanni da Montercorvino, Andrea da Perugia, Odorico da Pordenone e Giovanni de' Marignolli (ibid.). Se sinora sono stati citati viaggiatori occidentali, in massima parte italiani, che raggiunsero la Cina attraverso la Via della Seta, va però sottolineato come si sia trattato di un flusso umano con un doppio verso, il quale permise l'arrivo in Europa anche di viaggiatori sudditi del Gran Khan, come nel caso del monaco nestoriano Rabban Bar Sauma (Borbone 2000).

A partire dal 1368 l'avvento della dinastia Ming, etnicamente riconducibile agli Han, significò invece un deciso cambio di politica e un ripiegamento della Cina su se stessa: i traffici terrestri lungo la Via della Seta imboccarono una parabola discendente.

Diventato un itinerario marginale, poi un tema culturale declinato al passato e infine turistico, dopo molti secoli il concetto di Via della Seta è stato infine riattualizzato dal presidente cinese Xi Jinping con il lancio, nel 2013, del programma denominato One Belt One Road o Nuove Vie della Seta, ambizioso progetto infrastrutturale dalle evidenti connotazioni geopolitiche.

Ciò non significa ovviamente che nel lungo intervallo temporale tra l'instaurarsi della dinastia Ming e il XXI secolo siano venuti meno i viaggi tra la Cina e l'Occidente. I contatti continuarono a verificarsi, ma videro ora una netta prevalenza delle vie marittime rispetto a quelle terrestri.

Tra la fine del XVI secolo e il primo quarto circa del XVIII si materializzò infatti un nuovo incontro tra mondo cinese e mondo europeo, che vide come protagonisti economico-politici dapprima i portoghesi e come protagonisti culturali questa volta i gesuiti: entrambi gli attori impostarono però i propri flussi verso la Cina su navi oceaniche e su lunghe rotte marittime, e non su vie terrestri come in passato.

Dopo uno iato di circa un secolo connesso al bando della predicazione del Cristianesimo in terra cinese deciso dalla nuova dinastia mancese Qing, durante il quale la presenza europea venne quasi azzerata, a partire dalla fine degli anni Trenta del XIX secolo il Celeste Impero subì un vero e proprio assalto imperialistico occidentale, in primis britannico. Tale dinamica portò, tramite la stipula dei cosiddetti Trattati ineguali, alla creazione di un 
sistema para-coloniale in numerose città costiere cinesi (il caso più eclatante fu forse quello di Shanghai), destinato a perpetuarsi attraverso la Repubblica di Cina (1912) sino all'invasione giapponese e alla seconda guerra mondiale, per poi essere definitamente epurato dall'ascesa della Repubblica Popolare Cinese (1949). Di nuovo, tra Ottocento e Novecento i contatti e gli scontri sino-europei si svilupparono lungo vie marittime: in particolare, la transizione propulsiva dalla vela al vapore (metà circa del XIX secolo) e l'apertura del Canale di Suez (1869) permisero di abbreviare notevolmente i tempi di percorrenza dall'Europa all'Estremo Oriente.

Si colloca appunto nella stagione storica dell'imperialismo occidentale, al tramonto della dinastia Qing e poco prima della Rivoluzione Xinhai, il viaggio in Cina di Carla Novellis di Coarazze (1865-?). Di famiglia ungherese ed educata nel mondo mitteleuropeo tra Vienna e Monaco di Baviera, Carla Novellis di Coarazze (nata Dreyfus) completò, nel 1906-1907, un lungo viaggio in Estremo Oriente al seguito del marito, il barone Carlo Maria Novellis di Coarazze (1855-1916), al cui comando era stato affidato l'incrociatore della Regia Marina Italiana Marco Polo (denominazione quanto mai significativa circa la rilettura in chiave nazionalistica della figura del mercante veneziano) nel contesto di una missione destinata a toccare Corea, Giappone, le coste dell'impero zarista affacciate sul Pacifico, Cina, Hong Kong (Paoletti 2000; Rastelli 2011). Impossibilitata ad essere imbarcata con continuità su una unità militare, a tratti la Novellis seguì le rotte del coniuge su navi di linea, per poi ricongiungersi con lui nelle città in cui erano previste soste del Marco Polo nell'ambito della spedizione (Reina 2012).

Il viaggio della Nostra fu puntualmente annotato in un diario quasi giornaliero, scritto in un italiano leggermente stentato e intitolato $I I-\mathrm{Co}_{-}$ rea e Giappone ${ }^{1}$, tuttora inedito e oggi conservato presso l'Archivio Diaristico Nazionale di Pieve Santo Stefano (AR DV/90; Ricci 2003).

Il lavoro della Novellis verrà qui analizzato limitatamente alla sola sezione cinese (novembre 1906 - gennaio 1907), nell'ambito della quale ella trattò dei suoi soggiorni o delle sue visite a Shanghai, Suzhou e Canton, discutendo le descrizioni delle aree urbane e offrendo alcune proposte di identificazione di singoli luoghi citati nei passi. Non saranno invece presi in considerazione, perché al tempo della Novellis non ricompresi all'interno del territorio cinese, gli allora possedimenti coloniali di Hong Kong e Macao, rispettivamente britannico e portoghese, a cui la Nostra dedicò diverse pagine.

1 Il numerale II ricompreso nel titolo sottintende evidentemente un primo diario dell'autrice relativo alla sezione del viaggio precedente all'arrivo in Estremo Oriente, ad ora però non rintracciato né versato presso l'Archivio Diaristico Nazionale. 
Il diario in oggetto costituisce un'opera interessante sotto molteplici punti di vista, in quanto, a differenza della maggior parte dei lavori odeporici coevi di ambito estremo orientale, scritta 'in presa diretta' e per uso personale, priva quindi di filtri (dalle pagine emerge ad esempio un genuino entusiasmo per i luoghi che andava visitando) o di una rielaborazione ex post in chiave letteraria o pubblica. Altro elemento atipico risiede nel fatto che tale diario sia stato curato da una donna, quando invece la stragrande maggioranza dei racconti di viaggio dell'epoca furono redatti da uomini: nell'opera della Novellis è perciò rintracciabile una prospettiva di genere (De Courten 2005), individuabile ad esempio nei lunghi passi dedicati alla vita mondana nei circoli femminili di occidentali che ella man mano incontrava in Estremo Oriente, nell'interesse per la condizione della donna in Cina, oppure nell'assenza di accenni al 'mito nero' dei bordelli shanghaiesi, tra i maggiori del mondo nei primi decenni del Novecento, viceversa celebrati pochi anni dopo da Giovanni Comisso (1949) ${ }^{2}$.

Spontaneità e prospettiva di genere femminile accomunano il diario della Novellis a quello di un'altra viaggiatrice, Giuseppina Croci, quest'ultima trasferitasi a Shanghai dal 1890 al 1895 come assistente in una filanda di seta (Piastra 2014). I due racconti odeporici divergono però notevolmente se guardiamo al rispettivo background di riferimento: colta, poliglotta e abituata agli ambienti elitari la prima; appartenente alla classe lavoratrice e decisamente più ingenua nella sua visione della Cina la seconda.

Sullo sfondo del diario della Novellis emergono nitidamente le dinamiche quasi-coloniali del tempo in terra cinese: l'interazione dell'autrice si riduce in pratica alla sola comunità internazionale delle città che va visitando, e in modo preferenziale a quella italiana; i suoi rapporti con i residenti cinesi sono invece ridottissimi, e si limitano in massima parte, tranne rare eccezioni, alle classi subalterne coinvolte nei trasporti oppure nel facchinaggio (coolies, conduttori di risciò, ecc.), o a negozianti presso cui acquistare compulsivamente 'cineserie', assecondando un gusto per l'esotico e per il collezionismo tipico del periodo.

\section{Shanghai}

Carla Novellis di Coarazze giunse da sola a Shanghai, dove si sarebbe ricongiunta al marito, a bordo di una nave di linea del Norddeutscher Lloyd proveniente dal Giappone: l'uso da parte sua di un vapore tedesco va in-

2 Quest'ultima opera è però basata su viaggi dell'autore risalenti ai decenni precedenti. 
quadrato nel più ampio contesto della totale assenza, all'epoca, di linee di navigazione italiane stabili in Estremo Oriente; solamente nel 1919 tale lacuna sarà colmata dal Lloyd Triestino (Surdich 2016), compagnia destinata a divenire in breve tempo leader del trasporto passeggeri dall'Europa alla Cina e al Giappone fra anni Venti e Trenta del Novecento.

La Novellis, dopo una sosta intermedia della sua nave a Wusong, porto fluviale satellite rispetto a Shanghai, ubicato alla confluenza tra Fiume Azzurro e Huangpu e tappa fissa delle rotte (Piastra 2016a), arrivò in città il 15 novembre 1906, per rimanervi sino all'11 gennaio 1907: si trattò del soggiorno più lungo in terra cinese da parte dell'autrice e, di riflesso, della città del Celeste Impero a cui è dedicato di gran lunga il maggior numero di pagine.

La Shanghai visitata dalla Nostra è quella che è oggi nota come 'Vecchia Shanghai' ('Old Shanghai' in inglese; 'Vieux Shanghai' in francese; 老上海 'Lao Shanghai' in cinese), un'espressione che nella letteratura scientifica individua l'irripetibile stagione di espansione sperimentata da tale area urbana a partire dalla sua apertura al commercio internazionale in seguito al Trattato di Nanchino (1842) sino all'abolizione delle concessioni straniere qui impiantate, attuata dai giapponesi durante la seconda guerra mondiale. Si trattava di una metropoli tra le maggiori al mondo, cosmopolita e mercantilistica, articolata spazialmente, in una situazione per molti versi assimilabile a quella delle città coloniali, nella 'città vecchia' cinese (la Shanghai originaria), nell'International Settlement (sino al 1863, concessioni britannica e americana) e nella concessione francese (Fig. 1). Il mito edonistico della 'Vecchia Shanghai' diede ben presto vita, in Occidente, a stereotipi e banalizzazioni (la 'Parigi d'Oriente', la 'città del peccato', la 'città dell'oppio', la 'città in vendita', ecc.), tuttora in parte vivi.

A Shanghai la Novellis fu ospite del console italiano, Cesare Nerazzini ${ }^{3}$ (Francioni 2004), e delle personalità più in vista della comunità del nostro paese, a partire dalle famiglie di Ernesto Ghisi e di Achille Riva, più volte menzionate nello scritto, da tempo insediatesi in città e appartenenti a un flusso migratorio specializzato proveniente dal Milanese e imperniato sulla seta, entro cui rientrava anche l'esperienza della già menzionata Giuseppina Croci, tipico dell'ultimo quarto del XIX secolo (Piastra in corso di stampa). L'autrice visitò soprattutto l'International Settlement shanghaiese, posto sotto l'egida anglosassone, a partire dalle vie più centrali, famose per lo shopping.

3 La Novellis si trovò a Shanghai in occasione della fine del mandato consolare di Nerazzini, affermando di aver partecipato ai festeggiamenti per il suo commiato tra gli ultimissimi giorni del 1906 e i primissimi del 1907. 


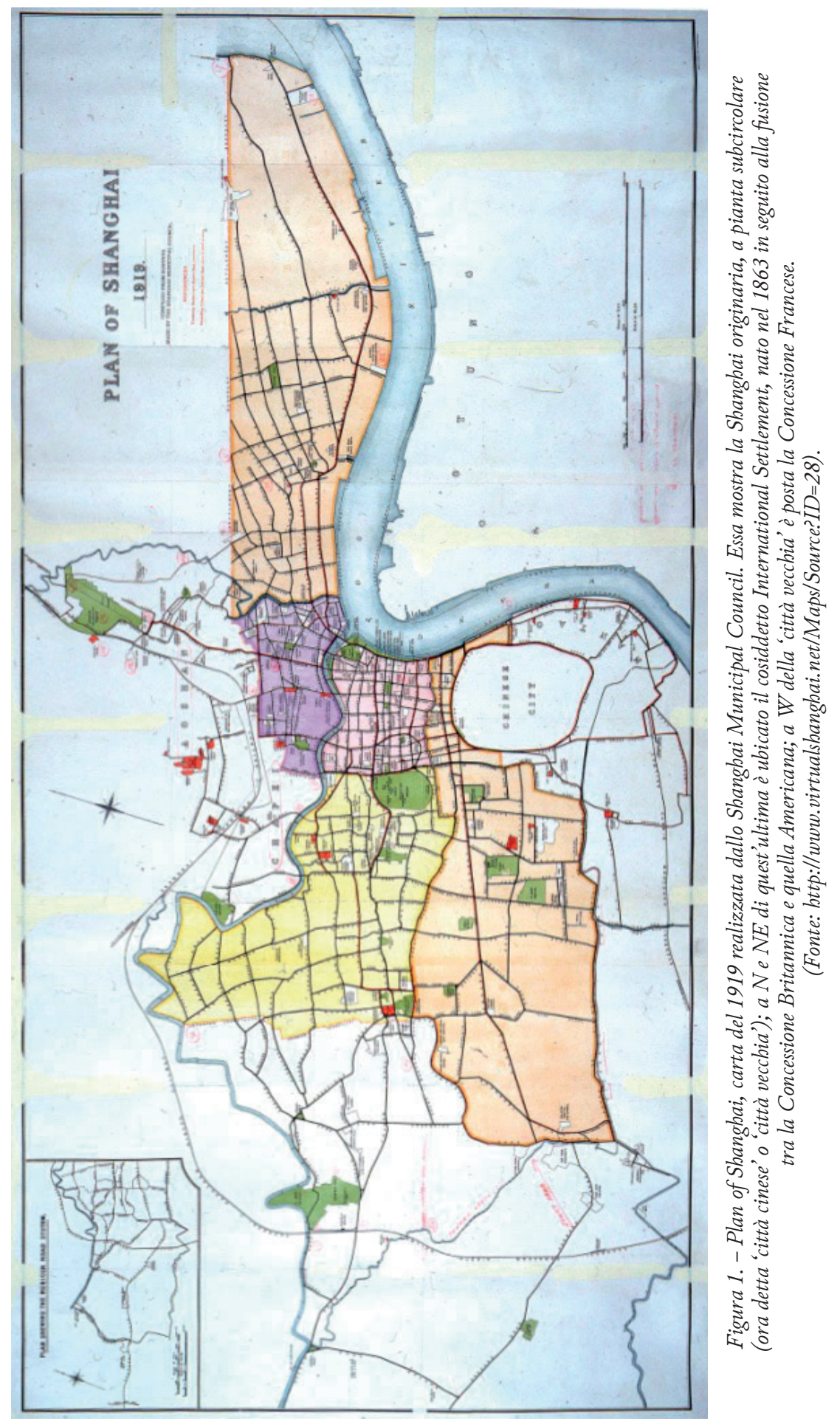


È il caso di Nanking Road ${ }^{4}$ (oggi East Nanjing Road), limitrofa al Bund, il famoso porto fluviale shanghaiese, di cui sottolinea l'affollamento e l'atmosfera cosmopolita ${ }^{5}$ :

Oggi ho fatto tutta Nanking-Road su e giù a piedi; è veramente un piacere. Quelle belle case cinesi colle insegne di legno scolpito e dorato, i negozi, la gente di tutti i generi. Europei e Cinesi molto per bene, mandarini in carrozza o portantine, gente bassa sui wheel-barrow (carretti con una ruota) molti rickshaw [risciò] carrozze signorili col cocchiere e boy [camerieri o collaboratori domestici cinesi] in livrea, Tartara [mancesi], Signore a cavallo, signore cinesi in coùpes tutto di vetro, automobili in quantità. Ad ogni angolo le guardie indiane coi turbanti rossi in testa. Una vita ed un'animazione come a Roma sul corso. [...] Poi molte piccole portantine con uomini e donne dentro, servitori a cavallo, tutti vestiti stranamente con berretti altissimi. Era un bellissimo colpo d'occhio.

Nel testo, il riferimento a "guardie indiane" rimanda ai poliziotti sikb dell'International Settlement, specificamente arruolati dallo Shanghai Municipal Council.

La Novellis descrive poi Canton Road (oggi Guangdong Road), posta all'estremità meridionale dell'International Settlement shanghaiese, nei pressi del suo confine con la concessione francese:

[...] il Signor Ricciardi [un membro della comunità italiana a Shanghai, che intrattiene in città i Novellis] [...] ci ha accompagnato a casa a piedi passando Canton-Road e diverse altre strade laterali, completamente cinesi e così pittoreschi che ogni momento pareva di vedere un quadro. Negozi d'argento cinese in quantità, botteghe dove si fabbricava tutti gli oggetti di carta, che bruciano ai funerali, casse, portantine, cavalli, gioielli, braccialetti, corone da sposa, ecc. Ma fatto così bene che paiono oggetti veri, soltanto guardando da vicino si vede che sono di carta. [...] Poi una gran quantità di brick a brack, uno vicino all'altro e per Carlo [Novellis di Coarazze, marito della Nostra] era una tentazione grande, non ha potuto fare a meno di comperare un vaso per 20 centesimi. Non ci siamo rovinati.

4 L'odonomastica dell'International Settlement della 'Vecchia Shanghai', coerentemente con il sistema quasi-coloniale della concessione posto sotto l'egida anglosassone, era in lingua inglese. Nel caso di traslitterazioni di nomi o toponimi cinesi, essi seguivano il sistema Wade-Giles.

5 In questa sede si riporta fedelmente il diario della Novellis, mantenendone grafie, punteggiature ed eventuali errori ortografici o grammaticali. Correzioni o integrazioni sono riportate entro parentesi quadre. 
Al di là dello shopping, la Novellis visita alcune curiosità urbane shanghaiesi, come la zecca di tael d'argento, monete cinesi allora in corso, posta in Peking Road (oggi East Beijing Road), poco più a sud del Suzhou Creek:

Ieri Gravina [Manfredi Gravina di Ramacca ${ }^{6}$, altro accompagnatore italiano della Novellis in città] ed io siamo stati [...] alla fonderia di tael in Peking Road. È tutto un quartiere di banquieri cinesi della Provincia del Shansi e vi sono milioni e milioni, tutti in grossi pezzi d'argento di 50 taels. [...] Le case hanno l'aria modestissima e nei cortili quasi in strada fondano l'argento per fare i tael.

La Novellis descrive anche l'arrivo del Marco Polo, con a bordo il marito, in città:

Arrivato verso il giardino pubblico sul Bund se lo vedeva fare il giro del fiume Whangpoo [Huangpu] e nel momento che gettava la ancora [...]. Poi è venuta la barca a vapore a prendermi e con Gravina sono andata a bordo, felice e contenta di vedere il 'Marco Polo' sano e salvo è successo niente di tutto quello che temevo.

Il "giardino pubblico" presso il Bund dove la Nostra osserva l'attracco del Marco Polo va identificato con il cosiddetto Public Garden shanghaiese, ovvero l'odierno Huangpu Park, da parte comunista cinese assurto a uno dei simboli dell'imperialismo occidentale in Cina prima dell'avvento della Repubblica Popolare, in quanto interdetto ai cinesi a cavallo tra XIX e XX secolo (Piastra 2016a). Ma durante il proprio soggiorno a Shanghai l'autrice si avventurò anche al di fuori dell'International Settlement, visitando la 'città vecchia' cinese:

Anche oggi abbiamo fatto ancora un giro nella città cinese, entrando da un'altra porta e traversandola tutta per sortire dalla porta dell'acqua [la Grande Porta Orientale della 'città vecchia', prossima al fiume Huangpu?]. Quante cose interessanti! La nostra guida era Francois [nome francese di un non meglio precisato uomo cinese], un ricco compradore ${ }^{7}$, cattolico e veramente perché già da 11 generazioni la sua famiglia è cattolica. Ci ha fatto vedere anche una bella chiesa cattolica in centro della città che è fabbricata come un tempio buddista, colla grande porta d'entrata, il cortile, il tetto della forma caratteristica cinese coi lavori di legno ed i soliti ornamenti. Solo in cima c'è una grande croce [cf. Piastra 2016b, fig. 8]. Anche l'interno è come un tempio

6 Manfredi Gravina di Ramacca, ufficiale di Marina e già a bordo dell'incrociatore Marco Polo, fu distaccato a Shanghai dal 1905 al 1907 (Francioni 2004). Rientrato in Italia, diede alle stampe un importante volume sulla sua esperienza cinese: Gravina di Ramacca 1907.

7 Italianizzazione della Novellis di comprador, termine mutuato dal portoghese e utilizzato in inglese per indicare, durante la stagione imperialistica, agenti o personale direttivo cinesi che lavoravano per gruppi occidentali o nell'intermediazione di affari. 
colle belle striscie di seta ricamate ed ornamenti cinesi [cf. Piastra 2016b, fig. 9]. Vi è un presepio ${ }^{8}$ ben fatto con bei fiori freschi; un altare della $\mathrm{Ma}-$ donna di Pompei ed ancora due altri. Il tutto molto bello e molto ben tenuto; diverse chiese da noi potrebbero prendersi come modello quella povera piccola chiesa in mezzo alla grande città cinese. Il prete è cinese, ma parla benissimo francese ${ }^{9}$, proprio coll'accento parigino. È stato educato dai gesuiti a Ziccawei [Zikawei, ovvero Xujiahui], che hanno qui vicino il grande osservatorio meteorologico ${ }^{10}$. Ha un bel giardinetto colle roccie artificiali e la Madonna di Lourdes. Ha anche la scuola per 80 allievi 'des classes primaires' come diceva, e proprio un convitto, dormono e mangiano là.

Tale chiesa cattolica all'interno della 'città vecchia' shanghaiese, dallo stile architettonico cinese, va identificata con certezza nel cosiddetto Lao Tang, letteralmente 'Chiesa Vecchia' (Piastra 2016b). Fondata verso il 1640 dal gesuita siciliano Francesco Brancati (1607-1671) grazie all'appoggio finanziario della famiglia dello shanghaiese Xu Guangqi (1562-1633), convertito al Cattolicesimo da Matteo Ricci (1552-1610), essa nacque dalla consacrazione di un preesistente edificio pubblico cinese (da qui l'aspetto orientale, e non occidentale, della struttura), per poi conoscere una storia travagliata attraverso i secoli: soppressa verso il 1730 e trasformata in un tempio del Dio della Guerra cinese; ri-consacrata in chiesa cattolica nel 1861 su pressione francese ${ }^{11}$; ancora soppressa in seguito all'avvento della Repubblica Popolare Cinese dopo il 1949.

La sua istituzione molto precoce da parte di Brancati riveste grande valore, perché la chiesa, tra XVII e XVIII secolo, funzionò sia come centro di evangelizzazione, sia come polo culturale, stampando libri di religione cattolica in lingua cinese, ospitando un osservatorio astronomico nel campanile originario (il più antico centro di ricerca scientifica occidentale a Shanghai), offrendo alloggio a confratelli di Brancati; tra questi, Martino

8 La Novellis visita Shanghai proprio tra il Natale del 1906 e l'Epifania del 1907.

9 Evidentemente, durante questa sua visita alla 'città vecchia' accompagnata dal comprador Francois, la lingua franca usata dalla Novellis fu il francese, e non l'inglese o tantomeno l'italiano.

10 L'autrice ricorda esplicitamente tale osservatorio poiché a quel tempo forniva previsioni meteorologiche e informazioni sui tifoni per il Mar Cinese orientale alle navi in navigazione; anche il Marco Polo usufrui di tali dispacci. Si ricorda inoltre che, a pochi anni di distanza dal viaggio della Novellis, a partire dal 1920 l'osservatorio meteorologico shanghaiese di Xujiahui iniziò a ospitare un gesuita italiano, Ernesto Gherzi, destinato a diventare in breve tempo il direttore di tale centro.

11 Non a caso, il prete cinese che reggeva la chiesa, incontrato dalla Novellis al tempo della sua visita, parlava fluentemente francese e si era formato presso il centro gesuitico di Xujiahui, posto sotto l'egida francese. 
Martini (1614-1661), destinato a rivestire un ruolo primario circa la veicolazione della cultura e della geografia cinesi in Europa, il quale proprio in questo edificio intraprese lo studio del mandarino.

Tuttora conservato, sebbene rimaneggiato, sconsacrato e oggi, dopo un discutibile restauro degli ultimi anni, annesso a un centro sociale per anziani (Fig. 2), il Lao Tang costituisce la più antica chiesa oggi esistente in Cina (Zhou Jin 2014) e rappresenta il luogo shanghaiese per antonomasia dell'incontro tra cultura orientale e cultura occidentale: esso meriterebbe certamente una piena tutela e una riconversione a fini museali.

L'atipicità della struttura del Lao Tang colpì profondamente la Novellis, come si desume dal passo citato; il comprador Francois accompagnò la Nostra proprio qui verosimilmente in quanto cattolico e poiché desideroso di mostrarle, in chiave interculturale, un sito fondamentale della presenza cristiana occidentale, in primis italiana, in terra cinese.

Proseguendo nel corso della medesima visita alla 'città vecchia', l'autrice si imbatte in una seconda presenza architettonico-culturale da lei percepita come eccentrica:

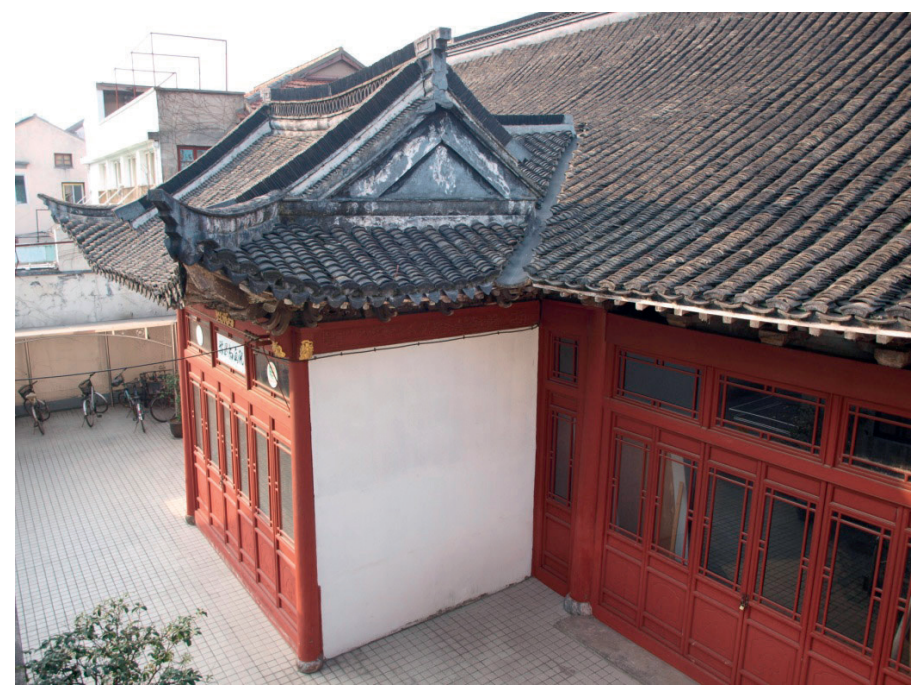

Figura 2. - 'Città vecchia' di Shanghai: il Lao Tang ('Chiesa Vecchia'), fondato verso il 1640 dal gesuita siciliano Francesco Brancati, cosi come appare oggi, dopo recenti

(e discutibili, in quanto gli infissi originali sono stati sostituiti) lavori di restauro. Attualmente l'edificio è annesso a un centro sociale per anziani; dato il suo grande valore culturale, esso meriterebbe una riconversione a fini museali.

(Foto S. Piastra, 2017). 
La cosa più strana che abbiamo avuto oggi era una Moschea. Una vera Moschea! Cioè anche il fabbricato di stile cinese ma dentro quasi tutto turco [ rectius, islamico]. La tavola coll'iscrizione, il pulpito, le stuoie in terra, la gente scalza, molte iscrizioni maomettane e quasi stuonavano i 'codini' ${ }^{12}$. Ma sono veri Maomettani [...]. Siamo rimasti colpiti dalla stranezza di vedere a pochi passi l'uno dall'altro un tempio di Budda, una moschea ed una chiesa cattolica.

Tralasciando lo stupore della Novellis circa l'imbattersi nel culto islamico in Cina (fatto insolito solo agli occhi di chi, come lei, non aveva alcun background orientalistico, vista l'esistenza di vastissime comunità musulmane specie nelle province nord-occidentali), appare verosimile un'identificazione della moschea citata dalla Nostra con quella oggi nota come Moschea di Fuyou Road oppure Moschea settentrionale, fondata nel 1870 e tuttora esistente (Fig. 3): rimandano a una tale interpretazione la sua vicinanza spaziale al Lao Tang e un aspetto esterno di gusto vagamente orientale non esplicitamente islamico. Non può invece trattarsi della Moschea Xiaotaoyuan, attualmente massimo luogo di culto musulmano cittadino e anch'essa ubicata nella 'città vecchia', ma istituita nel 1917, quindi successivamente al viaggio della Novellis.

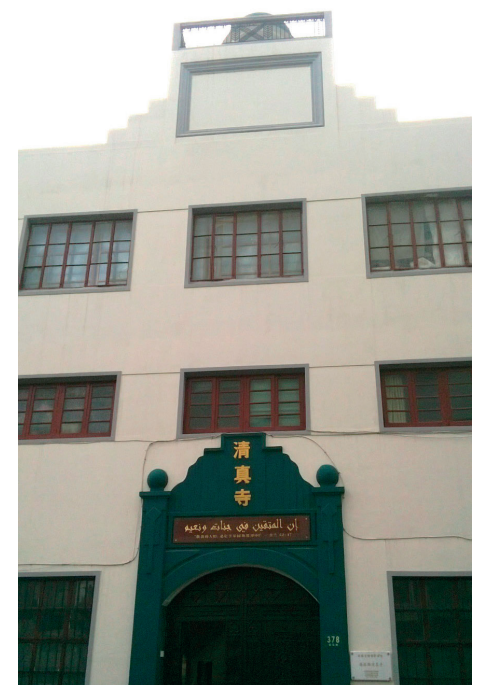

Figura 3. - 'Città vecchia'di Shanghai:

la Moschea di Fuyou Road.

(Foto S. Piastra, 2011).

12 La Novellis si riferisce all'acconciatura maschile di derivazione mancese tipica del periodo Qing, cancellata dal nuovo corso repubblicano cinese pochi anni dopo la sua visita. 
In relazione al "tempio di Budda" ricordato dall'autrice nei pressi del Lao Tang e della Moschea di Fuyou Road, non è da escludere che si possa trattare di un fraintendimento del Tempio del Dio della città di Shanghai, massimo edificio religioso cinese all'interno della 'città vecchia', a quel tempo molto popolare e ubicato non lontano da chiesa e moschea, scambiabile per un tempio buddista da parte di un osservatore non esperto di temi culturali e religiosi asiatici.

Un'ultima descrizione significativa è relativa a una passeggiata della Nostra lungo la sommità delle mura della 'città vecchia', edificate nel 1554 sotto la dinastia Ming per difendersi dalle incursioni di pirati giapponesi:

La mattina del 10 [gennaio 1907] quando io volevo mettermi a cominciare il bel lavoro dei bauli [in occasione della propria imminente partenza da Shanghai] ho mandato a domandare alla signora Ghisi [moglie di Ernesto, menzionato sopra] se vogliamo fare il giro delle mura della città cinese. Dunque partenza dopo le 10 ed in un'ora e mezza l'abbiamo fatto benissimo ed era veramente bello ed interessante. Dopo entrata la porta si sale su una rampa e di là si può fare il giro senza interruzione lungo i merli. Come tutte le mura cinesi è piuttosto un 'rampart' lungo di terra, foderato della parte esteriore di un muro e coronato con merli. Le porte mai dritte, aprendosi in una strada come da noi, ma sempre doppie formando un angolo di quasi 90 gradi, molto più difficile di prendere in caso d'assedio. [...] Io che credevo tutta popolata la città [vecchia] sono rimasta meravigliata di vedere molti orti e campi fra le case ed in certi quartieri poveri era una vera desolazione vedere le casette di legno che parevano di crollare, tutte storte e vicinissime proprio fra le case ed in mezzo gli orti le miserabili casse dei morti, certe nemmeno chiuse bene. I bambini qualche volta in semplici casse di petrolio. E tutte messe così in disordine in giro, secondo il consiglio degli astronomi [in realtà, i geomanti cinesi]. [...] Molti tintori erano al lavoro lungo le mura e distendevano le lunghe striscie di cottonata celeste ad asciugare al sole.

La testimonianza di dettaglio della Novellis circa le mura della 'città vecchia' risulta preziosa, in quanto tale struttura difensiva fu in massima parte demolita tra il 1912 e il 1913, in corrispondenza della transizione alla Repubblica di Cina, allo scopo di favorire le comunicazioni da/per il cuore storico urbano. Attualmente, sono pochissimi i lacerti di mura della 'città vecchia' shanghaiese sopravvissuti alla distruzione e ancora visibili, tra cui il Padiglione Dajing Ge.

Il riferimento finale alla lavorazione del cotone è significativo, riflettendo una delle vocazioni produttive storiche di Shanghai, riportata sin dalla metà del XVII secolo da Martino Martini (Piastra 2016a) e ripresa da Daniello Bartoli (Bartoli 1975). 


\section{Suzhou}

Il 30 dicembre 1906 Carla Novellis di Coarazze intraprese, partendo in treno da Shanghai, una gita di una giornata a Suzhou.

Si trattò di un'escursione estemporanea e brevissima, durante la quale la Nostra ebbe però l'occasione di confrontarsi con un paesaggio urbano più autenticamente cinese rispetto a quello di Shanghai, dove le concessioni occidentali riproponevano stilemi architettonico-urbanistici europei:

Dopo la porta [urbana] comincia la cosa più cinese vista fino ad ora, proprio non c'è niente di europeo. Le strade strettissime come le calli di Venezia, senza marciapiede, piene di gente e di vita, portantine, nobili a cavallo, molti altri su piccoli asini, una quantità di ponti con scalini (chiamano Soochov [Suzhou] la Venezia della Cina) poi un canale grande con un ponte ad arco quasi come il ponte di Rialto, pieno di venditori di tutti i generi.

Nel passo, la Novellis sottolinea la famosa definizione di Suzhou come 'Venezia della Cina' sulla base della diffusa presenza di canali urbani e ponti. In particolare, la menzione di una porta urbana a cui segue un canale attraversato da un imponente ponte arcuato sembra rimandare alla Porta Pan, in corrispondenza dell'angolo sud-occidentale urbano, e al vicino ponte Wumen, ancora esistente (Fig. 4), posto sul canale che corre attorno all'intero perimetro della città storica.

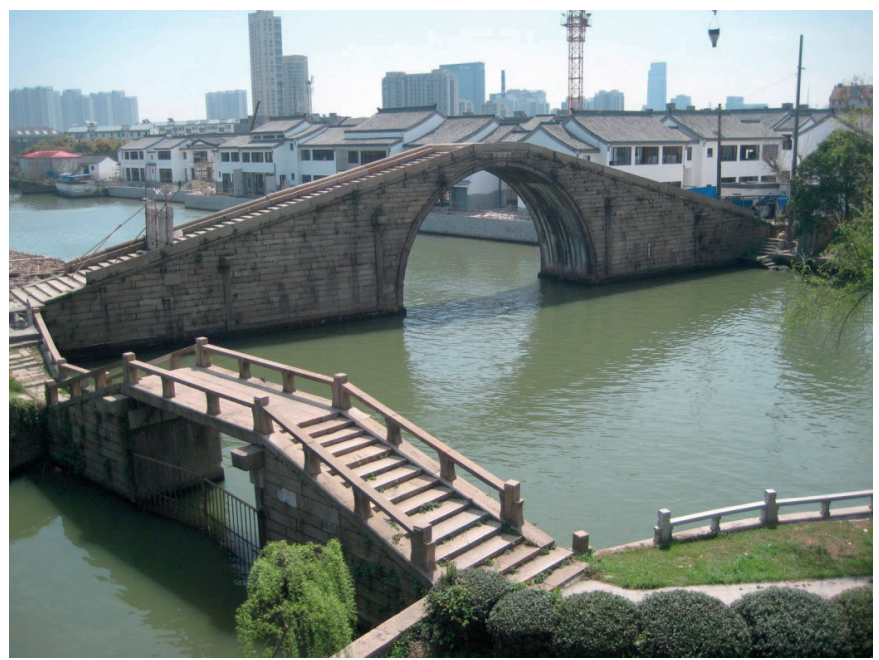

Figura 4. - Suzhou: il ponte Wumen. (Foto S. Piastra, 2013). 
L'uso del treno da parte dell'autrice per il suo spostamento da Shanghai a Suzhou alla fine del 1906 è riflesso degli sforzi di modernizzazione infrastrutturale del paese sul finire della dinastia Qing: pochi anni dopo, nel febbraio 1912, il giornalista italiano Salvatore Besso, partito sempre da Shanghai, avrebbe nuovamente percorso tale linea ferroviaria, ora prolungata sino a Nanchino, a quel tempo designata come capitale della neonata Repubblica di Cina (Piastra 2015).

\section{CAnton}

Alla fine di gennaio 1907 l'incrociatore Marco Polo faceva sosta nell'allora colonia britannica di Hong Kong. La Novellis ne approfittava per una breve gita di pochi giorni (24-26 gennaio 1907) con destinazione Canton, capoluogo della provincia cinese del Guangdong e anch'esso aperto al commercio internazionale, così come Shanghai, in seguito al Trattato di Nanchino (1842), risalendo su una nave a vapore l'estuario del Fiume delle Perle:

Del viaggio sul Canton-River (fiume Pearl) [il Fiume delle Perle] non mi sono accorta, perché la mattina svegliandomi eravamo già vicinissimi a Canton e si vedeva quella città immensa che si distende per molti chilometri e della quale non si sà se ha $1 \frac{1}{2}, 2$ o 3 milioni di abitanti. Molti vivono sulle loro barche, sampan grandi, dove mangiano, dormono, nascono, si sposano e muoiono. [...] I pochi passi dalla banchina fino alla piccola isola Shamin [Shamian], il settlement europeo abbiamo fatto a piedi. Shamin è tutto separato dalla città cinese da un canale ed i due ponti hanno porte di ferro e sono sempre guardate da soldati inglesi e francesi, i due proprietari [in realtà, concessionari] dell'isola. Ł̇ strettissima e solo in mezzo c'è una specie di strada [Central Avenue; vd. Fig. 5] che va da una punta all'altra e che si può fare in 10 minuti. Fra 2 o 300 europei arrivano qui, quasi tutti commercianti ed impiegati, ma c'è un club, negozi ed i villini sono carini e ben tenuti. [...] [Shamian] Non era che un banco di sabbia, lungo 600 metri e largo 200 ed è straordinario cosa ne hanno fatto gli inglesi ed i francesi. Un bel Bund con alberi alti in giro, un largo viale in mezzo e molti bei villini con giardini. Hanno acquedotto, pulizia, un tennis-club, danno pranzi e balli e si divertono ed hanno in mano tutto il commercio di seta.

L'autrice tratteggia dapprima la vastità dell'area urbana e accenna alle boat people cantonesi, per poi delineare l'isola di Shamian, circondata a sud dal Fiume delle Perle e a nord separata dalla terraferma da uno stretto canale: a partire dal 1859, quest'isola fu suddivisa in due concessioni (Fig. 5), 


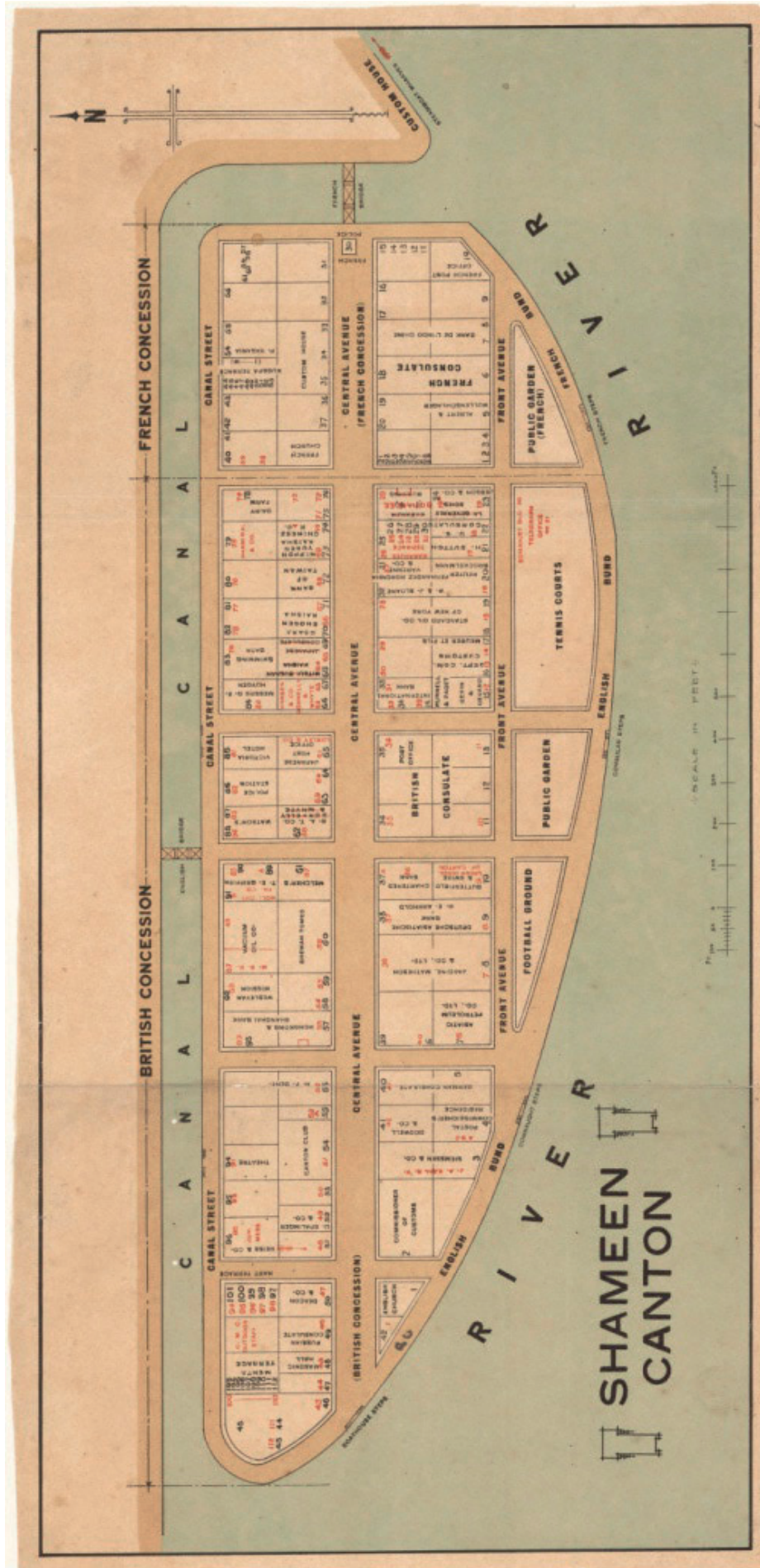

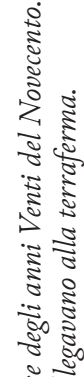

巡

: 02

ปี हิ

ป

ฏ ำ

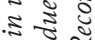

ค.

है เ

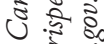

둘

है ङ

ชิ

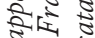

ङ2

离.

$\approx$ च

ई के ฮิ

क ल

ริ ปี

है

क०

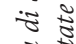

홍

곤

जे

定 
la più grande fu assegnata al Regno Unito e la più piccola alla Francia, divenendo una sorta di gated community ante litteram per gli europei qui residenti, in quanto gli unici due ponti che collegavano l'area alla città cinese al di là del canale (uno per concessione) di notte venivano chiusi ed erano continuamente presidiati da truppe sikb nella concessione britannica e vietnamite in quella francese. Gli espliciti riferimenti ad "acquedotto" e "pulizia" nelle concessioni rimandano al problema della sicurezza circa l'acqua potabile e al rischio-epidemie, temi quanto mai sensibili, ieri come oggi, in un ambiente di fatto tropicale e ad altissima densità umana come il Guangdong.

Passata quindi da Shamian alla Canton cinese, la Novellis menziona in modo rapido una "pagoda dei fiori", al cui interno pranzò assieme ad un suo accompagnatore italiano, di nome Penso, ex militare ora impiegato nelle dogane cinesi ${ }^{13}$ : si tratta del Tempio dei Sei Baniani, informalmente noto appunto come Pagoda dei Fiori.

Ritornata da Canton a Hong Kong, il 30 gennaio 1907 Carla Novellis di Coarazze lasciava definitivamente la Cina, sulla via del ritorno verso casa.

\section{RIFERIMENTI BIBLIOGRAFICI}

Bartoli, D. 1975. La Cina, a cura di B. Garavelli Mortara. Milano: Bompiani.

Bertuccioli, G., e F. Masini. 1996. Italia e Cina. Roma - Bari: Laterza.

Borbone, P.G. 2000. Storia di Mar Yabballaba e di Rabban Sauma. Un orientale in Occidente ai tempi di Marco Polo. Torino: Zamorani.

Comisso, G. 1949. Amori d'Oriente. Milano: Longanesi.

De Courten, L. 2005. "La cultura femminile dell'imperialismo. Il caso dell'Estremo Oriente". Giornale di storia contemporanea 8 (1): 7-26.

Francioni, A. 2004. Il 'banchetto cinese'. L'Italia fra le treaty powers. Siena: Nuova Immagine.

Gravina di Ramacca, M. 1907. La Cina dopo il millenovecento. Milano: Treves.

Paoletti, C. 2000. La Marina italiana in Estremo Oriente, 1866-2000. Roma: Ufficio Storico della Marina Militare.

Piastra, S. 2013. "La comunità italiana nella 'Vecchia Shanghai'. Temi socio-economici e di geografia urbana”. In Cina e Occidente. Incontri e incroci di pensiero, religio-

13 Quella qui delineata per Penso fu una traiettoria professionale comune a diversi italiani in Cina tra fine Ottocento e anni Trenta del Novecento, stabilitisi in particolare a Shanghai: vedi i casi discussi in Piastra 2013. 
ne e scienza, a cura di I. Doniselli Eramo e M. Sportelli, 69-92. Milano: Centro di Cultura Italia-Asia.

Piastra, S. 2014. "Il diario di Giuseppina Croci. La 'Vecchia Shanghai' in un racconto di viaggio atipico". In Un diario, molte storie. Il racconto di viaggio di Giuseppina Croci tra coordinate storico-geografiche e aspetti testuali, a cura di S. Piastra, 17-53. Udine: Forum.

Piastra, S. 2015, "Da 'necropoli' a capitale. Nanchino nella letteratura di viaggio italiana (1864-1937)”. Storia Urbana 146: 69-93.

Piastra, S. 2016a. "Gli occhi del diplomatico, gli occhi del naturalista. Percezioni della Cina nelle relazioni parallele di Vittorio Arminjon ed Enrico Hyllier Giglioli”. Sulla via del Catai 9 (15): 29-45.

Piastra, S. 2016b. "Francesco Brancati, Martino Martini and Shanghai's Lao Tang (Old Church). Mapping, Perception and Cultural Implications of a Place”. In Martino Martini (1614-1661), Man of Dialogue. Proceedings of the International Conference, Trento, October 15-17, 2014, edited by L.M. Paternicò, C. von Collani, and R. Scartezzini, 159-181. Trento: Università degli Studi di Trento.

Piastra, S. In corso di stampa. "Gli esordi e gli sviluppi dell'emigrazione italiana nella 'Vecchia Shanghai'. I nessi tra settore serico, origine lombarda, reti relazionale e familiare”. Bollettino della Società Geografica Italiana.

Rastelli, A. 2011. Italiani a Shanghai. La Regia Marina in Estremo Oriente. Milano: Mursia.

Reina, L. 2012. "Villaurea e le altre. Racconti di italiane in Oriente”. In Spazi segni parole. Percorsi di viaggiatrici italiane, a cura di F. Frediani, R. Ricorda, e L. Rossi Frediani, 145-168. Milano: FrancoAngeli.

Ricci, L., a cura di. 2003. Archivio Diaristico Nazionale. Inventario. Roma: Istituto Poligrafico e Zecca dello Stato.

Surdich, F. 2016. "Le rotte per la Cina tra XIX e XX secolo". Sulla via del Catai 9 (15): 73-83.

Zhou, J. 2014. Shanghai Church. Shanghai: Tongji University Press.

\section{Fonti inedite}

Archivio Diaristico Nazionale di Pieve Santo Stefano (AR), Carla Novellis di Coarazze, II - Corea e Giappone, DV/90.

Si ringraziano l'Archivio Diaristico Nazionale di Pieve Santo Stefano (AR) e i discendenti di Carla Novellis di Coarazze per aver permesso lo studio del racconto di viaggio oggetto del contributo. 
